\title{
A septo-temporal molecular gradient of sfrp3 in the dentate gyrus differentially regulates quiescent adult hippocampal neural stem cell activation
}

\author{
Jiaqi Sun ${ }^{1,2+}$, Michael A. Bonaguidi ${ }^{2,3,8+}$, Heechul Jun ${ }^{4}$, Junjie U. Guo ${ }^{2,3,9}$, Gerald J. Sun ${ }^{2,5}$, Brett Will2, \\ Zhengang Yang ${ }^{6}$, Mi-Hyeon Jang ${ }^{4}$, Hongjun Song ${ }^{2,3,5}$, Guo-li Ming ${ }^{2,3,5,7^{*}}$ and Kimberly M. Christian ${ }^{2,3}$
}

\begin{abstract}
Background: A converging body of evidence indicates that levels of adult hippocampal neurogenesis vary along the septo-temporal axis of the dentate gyrus, but the molecular mechanisms underlying this regional heterogeneity are not known. We previously identified a niche mechanism regulating proliferation and neuronal development in the adult mouse dentate gyrus resulting from the activity-regulated expression of secreted frizzled-related protein 3 (sfrp3) by mature neurons, which suppresses activation of radial glia-like neural stem cells (RGLs) through inhibition of Wingless/INT (WNT) protein signaling.

Results: Here, we show that activation rates within the quiescent RGL population decrease gradually along the septo-temporal axis in the adult mouse dentate gyrus, as defined by MCM2 expression in RGLs. Using in situ hybridization and quantitative real-time PCR, we identified an inverse septal-to-temporal increase in the expression of sfrp3 that emerges during postnatal development. Elimination of sfrp3 and its molecular gradient leads to increased RGL activation, preferentially in the temporal region of the adult dentate gyrus.

Conclusions: Our study identifies a niche mechanism that contributes to the graded distribution of neurogenesis in the adult dentate gyrus and has important implications for understanding functional differences associated with adult hippocampal neurogenesis along the septo-temporal axis.
\end{abstract}

Keywords: Adult hippocampal neurogenesis, Molecular gradient, neural stem cells, Septo-temporal axis, Wnt, niche

\section{Background}

Along with its well-known role in learning and memory, the hippocampus is a neural structure involved in the regulation of motivational behaviors, emotional states and stress responses [1,2]. The rodent hippocampus is an elongated, laminated structure with its long axis extending rostro-dorsally from the septal nuclei of the basal forebrain, over and behind the thalamus, and then caudo-ventrally to the temporal lobe. This longitudinal

\footnotetext{
* Correspondence: gming1@jhmi.edu

${ }^{\dagger}$ Equal contributors

${ }^{2}$ Institute for Cell Engineering, Johns Hopkins University School of Medicine, Baltimore, MD 21205, USA

${ }^{3}$ Department of Neurology, Johns Hopkins University School of Medicine,

Baltimore, MD 21205, USA

Full list of author information is available at the end of the article
}

axis of the hippocampus is usually referred to as the septo-temporal (or dorso-ventral) axis [3, 4]. Differences in anatomical connections and electrophysiological properties along the septo-temporal axis of the hippocampus have been well documented [5-11]. In addition, studies using targeted lesions and inactivation have revealed functional differences along this hippocampal axis. Specifically, the septal (dorsal) portion appears to be preferentially engaged in learning and memory processes associated with navigation and exploration, while the temporal (ventral) hippocampus appears to be more involved in mood and anxiety-related behaviors [12, 13]. Consistent with these findings, a recent study has demonstrated that acute activation of granule cells specifically in the dorsal or ventral hippocampal dentate gyrus 
differentially suppresses contextual learning or innate anxiety, respectively [14]. Moreover, region-specific gene expression supports segregation of the hippocampus into septal, intermediate and temporal zones [11, 15].

The adult mammalian hippocampus continuously generates new neurons that integrate into preexisting neuronal networks [16-18]. Adult neurogenesis is a complex process whereby quiescent radial glia-like neural stem cells (RGLs) give rise to newborn neurons that mature over several weeks [16-19]. Cumulative evidence suggests that several properties of adult neurogenesis vary throughout the longitudinal (septo-temporal) axis of the hippocampus. The magnitude of adult hippocampal neurogenesis is lower in the temporal region, as assessed by the number of RGLs, intermediate progenitor cells and immature neurons $[20,21]$. The pace of neurogenesis is also slower in the temporal region, but it appears to be more responsive to local niche dynamics and exogenous factors [22]. Not only can changes in neuronal activity alter the tempo of newborn neuron maturation more dramatically, but the age-related decline in precursor number occurs more rapidly in the temporal dentate gyrus [22-24]. Furthermore, studies indicate a functional dissociation for adult hippocampal neurogenesis along the longitudinal axis wherein newborn neurons in the dorsal dentate gyrus modulate learning of contextual discrimination under some conditions, while immature neurons in the ventral dentate gyrus mediate anxiolytic effects of the anti-depressant fluoxetine [25]. How these differences in neurogenesis along the longitudinal axis of hippocampus are regulated is a fundamental question that remains to be answered.

Morphogens and their expression gradients play an essential role in the patterning of organogenesis during embryonic development [26]. Among these, Wingless/ INT (Wnt) family members are critical morphogens that regulate numerous developmental processes, including neural stem cell maintenance and differentiation in the vertebrate central nervous system (CNS) [27, 28]. During embryonic and early postnatal development, Wnt signaling is essential for the proper formation of the hippocampus. For example, Wnt3a is expressed in the cortical hem, which serves as a signaling center for hippocampal development at the tip of the caudomedial cortical wall, and is crucial for the normal growth of the hippocampus [29]. Furthermore, fate specification is determined by the relative strength of Wnt signaling such that strong Wnt signaling biases differentiation toward a dentate granule cell fate, while moderate Wnt signaling specifies pyramidal cells of the cornus ammonis (CA) layers [30]. In the dentate gyrus of the adult mouse hippocampus, many Wnts and their inhibitors are expressed [31] and functional studies have revealed that Wnt signaling regulates multiple steps of adult hippocampal neurogenesis under different physiological conditions [32-39]. In particular, we recently showed that mature granule cells in the adult mouse dentate gyrus express secreted Frizzled-related protein 3 (sfrp3), a secreted inhibitor of Wnt signaling, and that deletion of sfrp3 leads to increased activation of quiescent RGLs and production of new neurons [32], as well as behaviors mimicking those observed with long-term antidepressant treatment [40]. In this study, we examined whether sfrp3 could contribute to differences in the magnitude of adult hippocampal neurogenesis along the septo-temporal axis.

\section{Results}

\section{Heterogeneity of quiescent RGL activation along the} septo-temporal axis of adult mouse dentate gyrus

Due to the curvature and orientation of the hippocampus (Fig. 1a), sectioning the brain in one of the three traditional planes (coronal, horizontal, sagittal) produces sections in which the septo-temporal coordinates are hard to define. To better visualize adult hippocampal neurogenesis along the septo-temporal axis, we first established a new method for sectioning. We fixed hippocampi dissected from the adult mouse brain and embedded them into O.C.T. mounting solution. The individual hippocampus was positioned such that coronal-like sections were obtained from much of the septo-temporal extent of the structure (Fig. 1a-b). For quantification, we divided the hippocampus into four regions along the septo-temporal axis (Fig. 1a-b). Using this approach, we first investigated whether a difference in the activation of quiescent RGLs exists along the septo-temporal axis of the hippocampus of adult mice. We identified RGLs as nestin ${ }^{+}$precursors localized within the sub-granular zone (SGZ) with radial processes extending towards the molecular layer [41] (Fig. 1c). We further used the expression of minichromosome maintenance complex component 2 (MCM2) as a marker for activation of largely quiescent RGLs since thymidine analogs such as EdU and BrdU are only incorporated during the $S$ phase of cell cycle and preferentially label intermediate progenitors and neuroblasts with a single injection [41-46] (Fig. 1c). Stereological quantification along the SGZ in four regions showed a gradual decrease in the density of $\mathrm{MCM}^{+}$RGLs along the septo-temporal axis of the hippocampus (Fig. 1c-d). These results confirmed previous findings of septo-temporal differences in adult hippocampal neurogenesis [20] and further identified a clear gradient in which activation of quiescent adult neural stem cells decreases along the septo-temporal axis.

\section{A gradient of sfrp3 expression along the septo-temporal axis of the adult dentate gyrus}

Next, we investigated the potential molecular niche mechanism underlying this heterogeneity in neurogenesis along the septo-temporal axis of the adult hippocampus. Several 


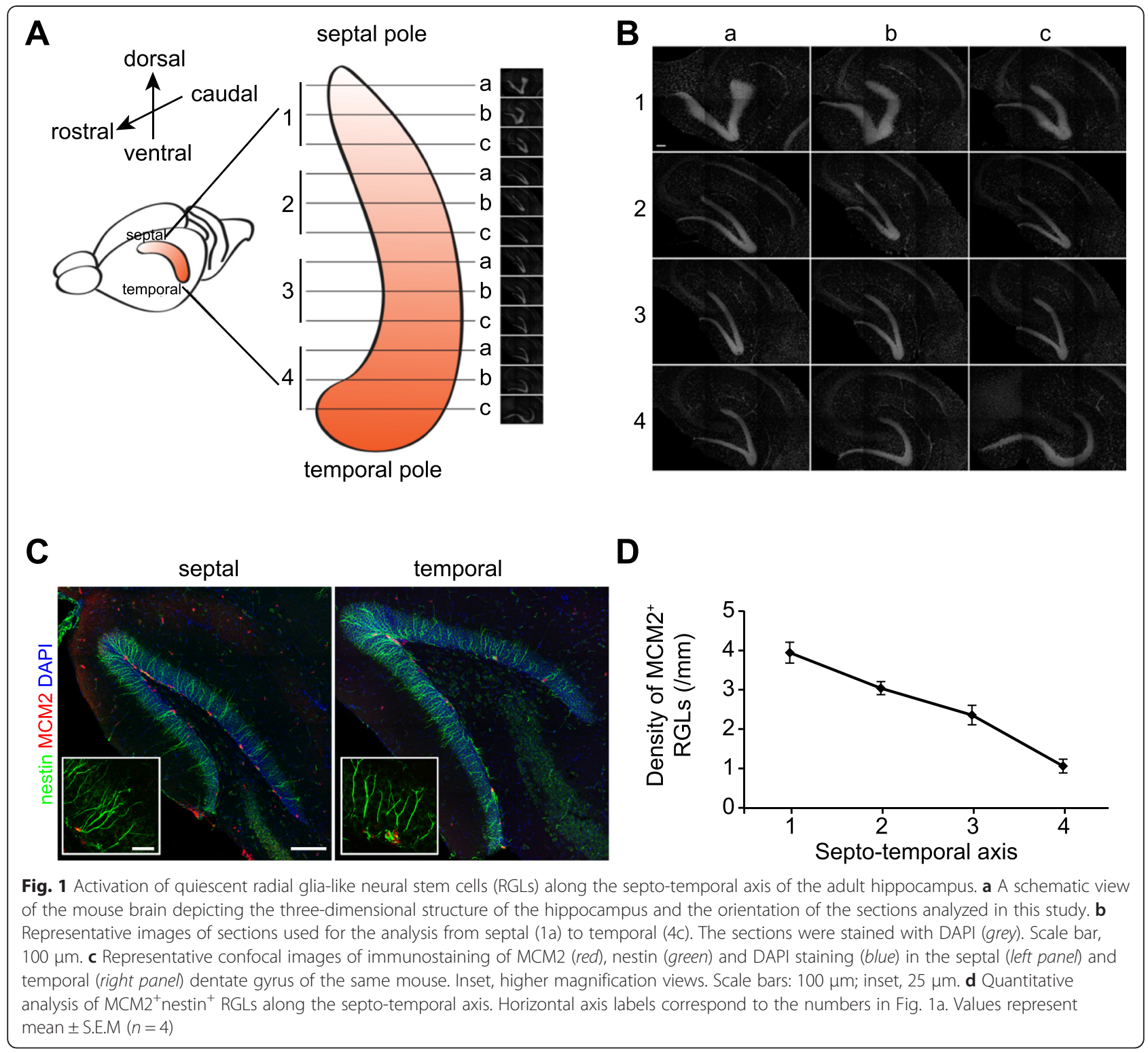

studies have revealed specific molecular domains and regionalization within the dentate gyrus $[11,15]$. We focused on sfrp 3 based on several of our recent findings. First, we found that in adult mice sfrp 3 is highly expressed in mature dentate granule cells and suppresses activation of quiescent RGLs and inhibits maturation of immature neurons [32]. Second, sfrp3 expression is downregulated by various antidepressant treatments, including electroconvulsive treatment and chemical antidepressants, whereas sfrp3 knockout mice exhibit behaviors resembling those following antidepressant treatment [40]. Third, we found that three single-nucleotide polymorphisms (SNPs) in the human SFRP3 gene are significantly associated with early antidepressant responses in a human patient cohort [40]. Given the proposed functional differentiation of the hippocampus along its long axis, we assessed whether the expression pattern of sfrp 3 aligns with this dissociation at the molecular level. We examined sfrp3 expression along the longitudinal axis of the adult dentate gyrus using in situ hybridization and quantitative real-time PCR analyses. To directly visualize sfrp 3 expression along the septo-temporal axis in a single section, we developed another new approach to section the dissected hippocampus in the sagittal plane (Fig. 2a). Consistent with previous reports [32, 47], sfrp3 expression was enriched in the granule layer of the adult dentate gyrus (Fig. 2a). Interestingly, the sfrp3 in situ signal intensity increased along the longitudinal axis of adult hippocampus from the septal pole to the temporal pole (Fig. 2a). Quantitative real-time PCR in samples of micro-dissected dentate gyrus further confirmed higher sfrp3 expression in the temporal dentate gyrus (Fig. 2b). Notably, in situ analysis showed that the sfrp3 

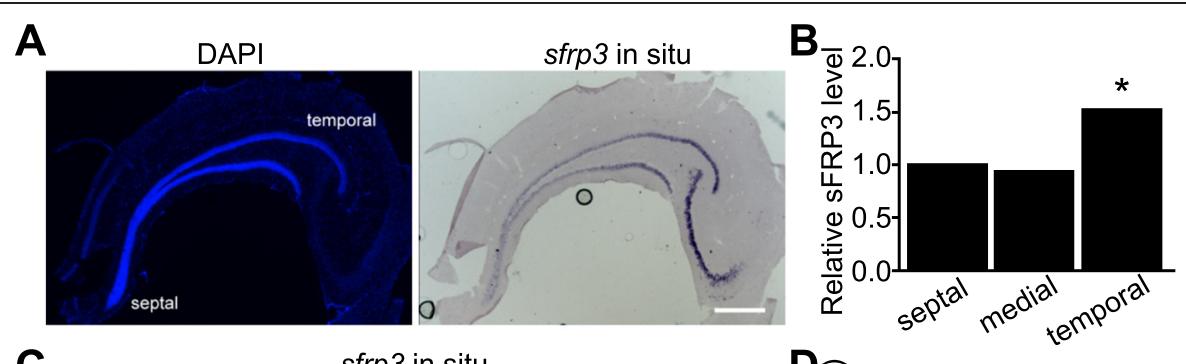

C

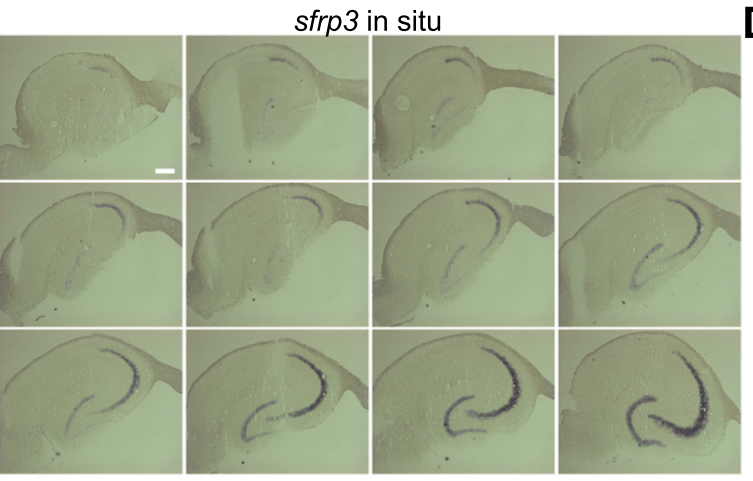

E

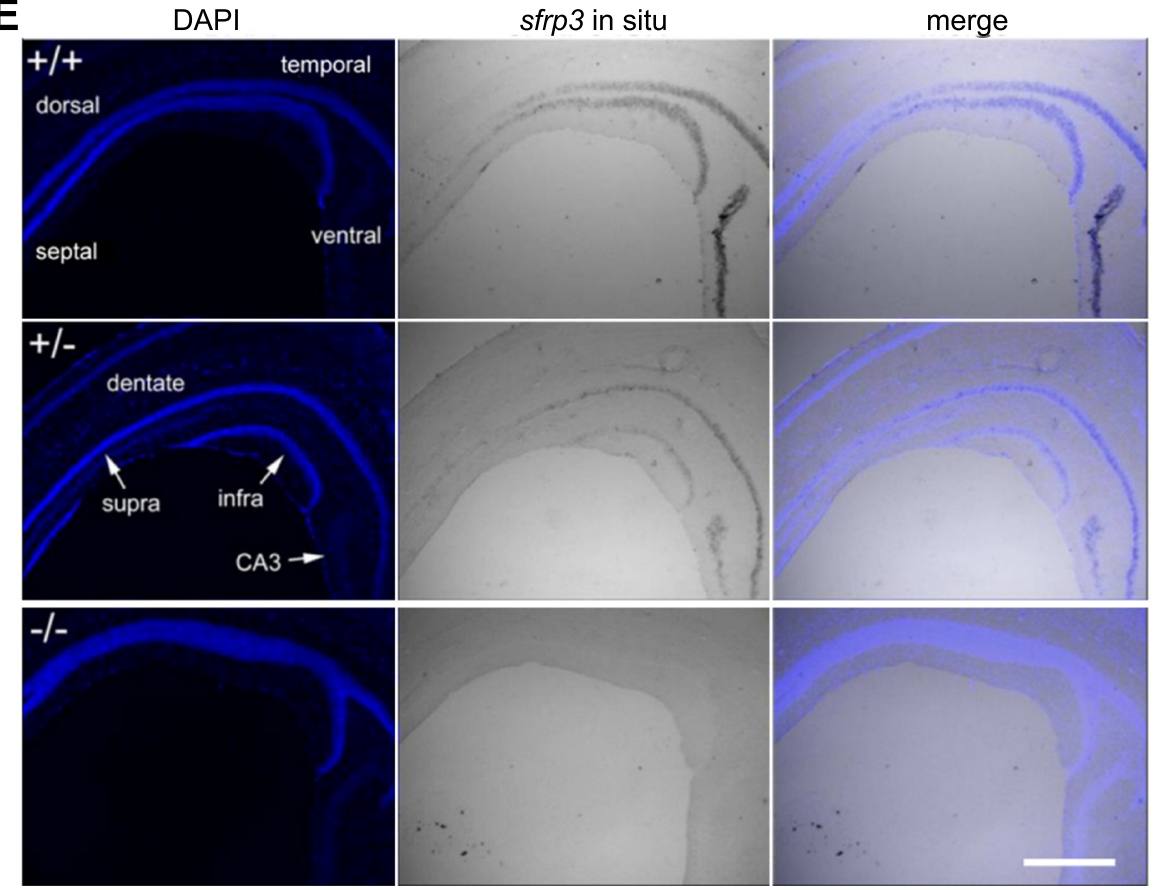

sfrp3 in situ

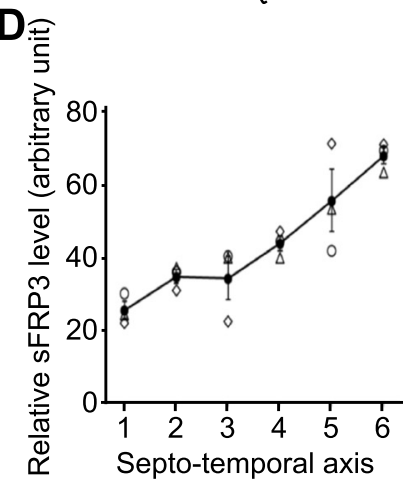

Fig. 2 A gradient of sfrp3 expression in the dentate gyrus of adult mouse hippocampus. a Mid-sagittal section of dissected mouse hippocampus showing in situ hybridization (ISH) for sfrp3 mRNA. Scale bar, $500 \mu \mathrm{m}$. b Quantitative analysis of sfrp3 expression by quantitative real-time PCR. Values present mean \pm S.E.M. $\left(n=4{ }^{*}<0.05\right.$; ANOVA). c. Representative images of ISH for sfrp3 mRNA on twelve serial sections along the septo-temporal axis, with the planes oriented as shown in Fig. 1a. Scale bar, $10 \mu \mathrm{m}$. d Quantification of signal intensity for sfrp3 in situ, which was performed on the images shown in Fig. 2c. Open circles, diamonds and triangles represent sfrp3 mRNA from septo-temporal axis of three individual mice. Closed circles are the mean of the three mice. Values represent mean \pm S.E.M. $(n=3)$. e Representative images of sfrp3 mRNA in situ in the dentate gyrus of adult sfrp3 knockout $\left({ }^{-/}\right)$, heterozygous $\left({ }^{+/-}\right)$, and wild-type $\left({ }^{+/+}\right)$littermates. Scale bar, $500 \mu \mathrm{m}$

gradient appeared to be continuous across the septotemporal axis of the hippocampus without sharp step changes suggestive of "border" transitions (Fig. 2a). We confirmed this result with densitometric measurements of in situ signal intensity on coronal sections of dissected hippocampus along the entire axis of hippocampus (Fig. 2c-d). To confirm the specificity of the sfrp3 in situ probe used, we compared wild-type $(+/+)$, heterozygous $(+/-)$ and knockout (-/-) sfrp3 animals. Indeed, no signal was observed in knockout animals in sections processed 
in parallel with other genotypes (Fig. 2e). Interestingly, a gradient was present in the heterozygous mice, although the expression level appeared to be reduced by $50 \%$ (Fig. 2e). Together, these results demonstrated that sfrp3 expression in the adult mouse dentate gyrus exhibits a smooth gradient with increasing expression along the septo-temporal axis.

\section{Developmental establishment of the sfrp3 expression gradient in the dentate gyrus}

Although it is well-known that morphogens act as graded positional cues to control cell fate specification in many developing tissues, the presence of a gradient in adult tissue is very rare. We next investigated the developmental time point when the gradient expression pattern of $s f r p 3$ is established. Previous studies have examined sfrp3 expression during early embryonic development. Specifically, sfrp 3 is not expressed in the cortical hem, which is the hippocampal organizer [48], but is instead expressed dorsal to this region at E14.5 and becomes confined to the dentate gyrus at E17.5 [49]. Using in situ hybridization, we examined the magnitude and time course of sfrp3 expression from postnatal day 7 (P7), when the dentate gyrus is recognizable as a morphological structure with two blades of discrete dentate granule cell layers (Fig. 3a). At P7, the sfrp3 in situ signal was scattered in the dentate granule cell layer and CA3. By P10, sfrp3 expression became prominent in the dentate gyrus, although still weaker than in the adult dentate gyrus. The gradient expression pattern was observed at P7, became readily apparent by P10, and persisted at P360 (Fig. 3a). Quantitative analysis showed that the slope of the sfrp 3 mRNA expression gradient increased between P7 and P56 (Fig. 3b). Together, these results revealed that the gradient expression pattern of sfrp3 is initiated during early postnatal stages and becomes more pronounced later and maintained during adulthood.

\section{Functional impact of sfpr 3 gradient on neurogenesis along the septo-temporal axis}

Finally, we examined the contribution of the sfrp3 molecular gradient on the differential regulation of quiescent RGL activation along the septo-temporal axis. Although it is impossible to rule out compensatory effects during development from germline transgenic mice, our previous analyses of adult sfrp 3 knockout mice did not indicate any gross or cumulative morphological effects on the dentate gyrus [40]. Consistent with our previous population study, sfrp3 knockout mice exhibited increased activation of quiescent RGLs as shown by the increased density of MCM2 ${ }^{+}$RGLs (Fig. 4a). This increase occurred in both septal and temporal regions and, notably, the magnitude of the change in quiescent RGL activation gradually increased from regions 1 to 3 along
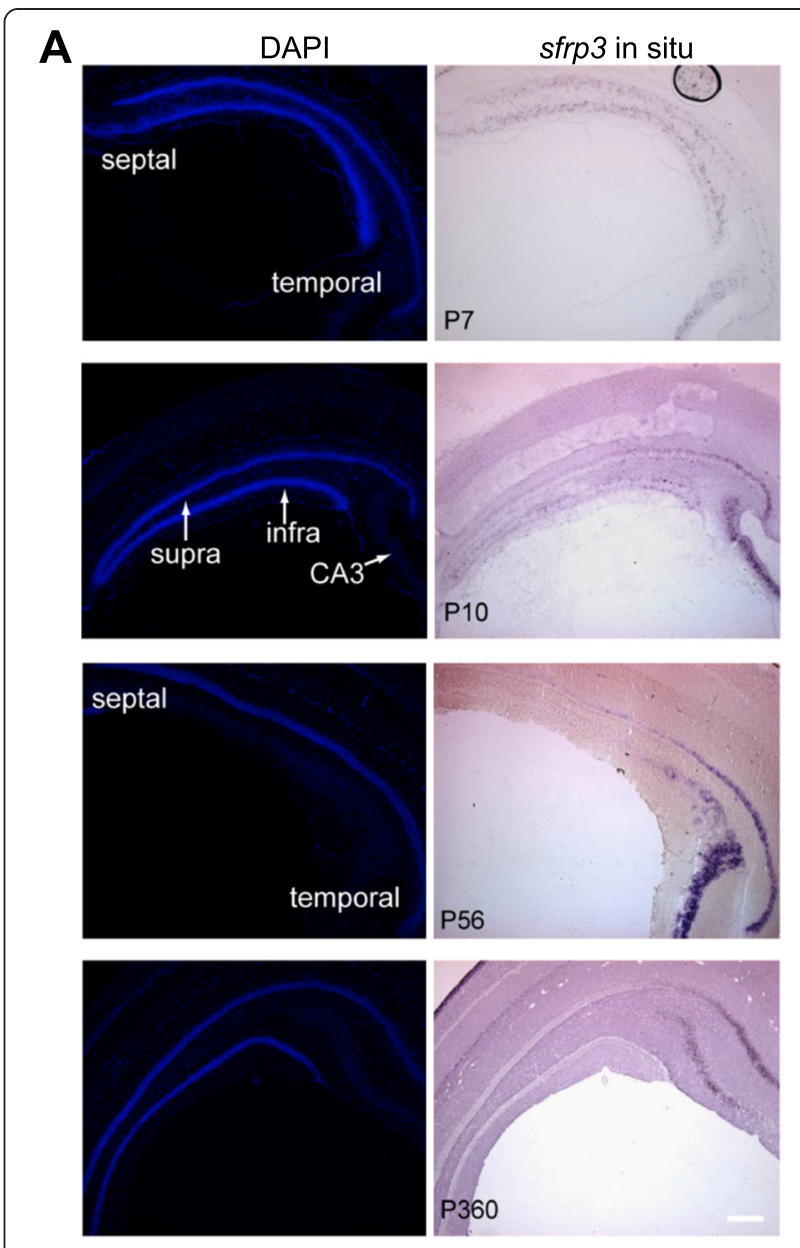

B

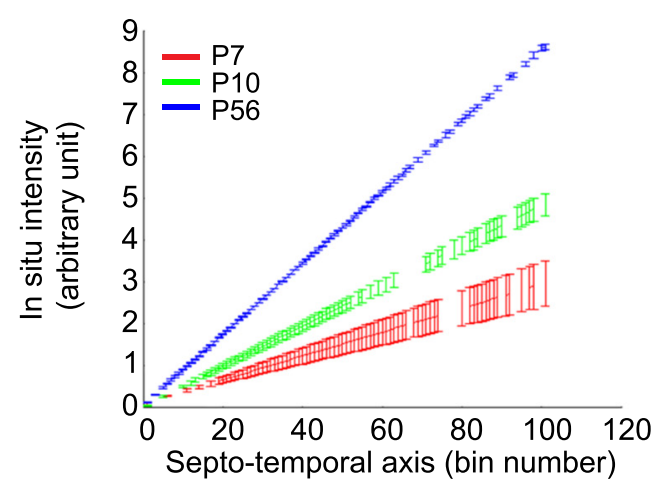

Fig. 3 A gradient of sfrp3 expression in dentate gyrus from early postnatal to aged animals. a Representative images of sfrp3 mRNA in situ in the dentate gyrus of wildtype mice at different ages (P7, P10, P56 and P360). Scale bar, $250 \mu \mathrm{m}$. b Quantification of signal intensity for sfrp3 in situ, which was performed on the images shown in Fig. 3a. Reconstructed dentate gyri from sagittal sections were divided into 100 bins (represented on the $X$ axis) for quantification of signal intensity along the septo-temporal axis. Values represent mean \pm S.E.M. $(n=3)$. The sfrp3 mRNA expression increases from septal to temporal dentate gyrus at each age from P7 to P56 in WT mice with an increase in slope during the interval of P7-P56 
the septo-temporal axis, but not in region 4 at the most temporal pole (Fig. 4b). Therefore, removal of sfrp3 expression and its associated gradient dampens, but does not completely abolish, the septo-temporal differences in quiescent RGL activation during adult hippocampal neurogenesis. These results support an active role for the sfrp3 gradient in differential regulation of stem cell activation along the septo-temporal axis, but also point to additional mechanism(s) that may mediate residual differences, particularly in the temporal pole.

To further support our model, we examined quiescent RGL activation using an independent approach via clonal lineage-tracing of individual quiescent RGLs. We previously established a clonal assay in which we could label individual quiescent RGLs in adult nestin-CreER ${ }^{T 2}$ ${ }^{+/-} ; Z / E G^{f /+}$ mice with injection of a single low dose of tamoxifen to quantify their activation rates and fate choices $[32,41,45]$. We generated adult nestin-CreER ${ }^{T 2}$ ${ }^{+/} ; \mathrm{Z} / E G^{f /+} ;$ sfrp ${ }^{-/-}$and nestin-CreER ${ }^{T 2+/-} ; Z / E G^{f /+} ;$ sfrp3 ${ }^{+/+}$mice and performed short-term clonal analysis. We quantified quiescent (an isolated RGL) or activated (an
RGL with one or more progeny in close proximity) individual clones with respect to their septal or temporal localization in the dentate gyrus (Fig. 4c), which indicated the cumulative RGL activation during the testing period. Consistent with previous studies [32], sfrp3 knockout mice exhibited an increase in the number of activated RGLs throughout the hippocampus (Fig. 4d). Notably, the increase in RGL activation is more than doubled in the temporal region compared to that in the septal region (Fig. 4e). These results also support our model that the graded sfrp3 expression along the longitudinal hippocampal axis contributes to the regional differences in RGL activation rates.

\section{Discussion}

In the developing brain, morphogen gradients are commonly used to pattern neurogenic regions into distinct functional domains. Examples of such gradients are WNTs that are responsible for specifying dorsal fates during telencephalic neurogenesis [50]. Several members of the Wnt family (including Wnt-2b, Wnt-3a, Wnt-5a,

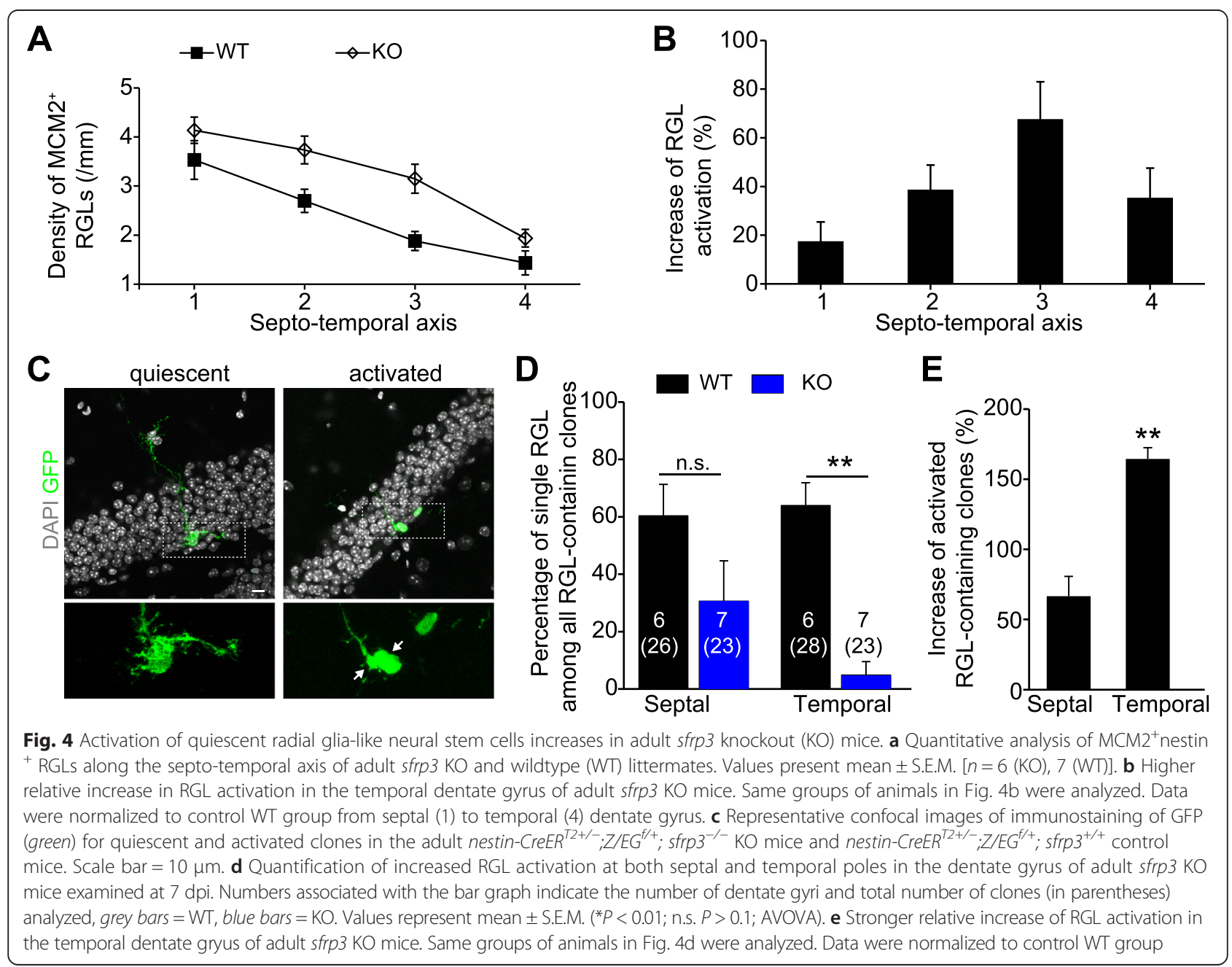


Wnt-7b, and Wnt-8b), as well as members of the SFRP family of Wnt inhibitors, are highly expressed in the cortical hem, a medial telencephalic signaling center, which is also the primary hippocampal organizer [28, 51]. Gradients of Wnt family members are well studied in the developing hippocampus [49]. Whether any of these gradients persist into adulthood was not previously known. Early in development, sfrp3 is expressed weakly in the medial neuroepithelium and medial non-neural mesenchyme, but is excluded from the cortical hem. Later, sfrp3 is expressed in cells from the dentate notch to the developing dentate gyrus along the migratory route of newly born dentate granule cells and their precursors. In addition, sfrp3 is expressed in the tertiary matrix, which is the displaced mitotic zone that transiently forms at the termination of this migratory route in the hilus of the dentate gyrus [49]. Here we identified a graded expression pattern of sfrp3 that emerges by postnatal day 7. Our study revealed, for the first time, that a morphogen-related protein gradient is generated postnatally and maintained in the adult mammalian brain.

The proliferation and integration of adult-born neurons into existing hippocampal circuitry has been implicated in a wide range of behaviors, including context discrimination, pattern separation, spatial learning, anxiety, and responses to antidepressants [18, 52-57]. These diverse functions may reflect differences in the local hippocampal network along the septal-temporal axis, with respect to anatomical connections and electrophysiological properties. For example, serotonergic fibers provide denser input to the temporal hippocampus with a concomitant enrichment of 5-HT1A and $2 \mathrm{C}$ receptors $[10,58]$. Long-term potentiation (LTP) is both larger and longer lasting in septal slices [59]. Growing evidence supports that adult neurogenesis in the dentate gyrus is also heterogeneous along this longitudinal axis. A higher number of proliferating cells and faster maturation rates occur in the septal dentate gyrus compared to the temporal region [24]. Because adult neurogenesis has been implicated in both cognitive and affective behaviors, an exciting possibility is that adult-born granule cells in the septal and temporal hippocampus may be functionally dissociated [25]. However, little was known about potential niche mechanisms that could contribute to septotemporal heterogeneity in hippocampal neurogenesis processes and properties. Our study identifies a gradient of expression of $s f r p 3$ (inferred from its mRNA levels), a niche factor secreted by mature dentate granule neurons, that results in differential control of neurogenesis along the longitudinal axis of the dentate gyrus. Both population analysis and in vivo clonal analysis, using a genetic sparse-labeling approach, indicate that removal of $s f r p 3$ and its gradient result in preferentially increased RGL activation in the temporal dentate gyrus. Since higher levels of sfrp3 are expressed under physiological conditions in this region, our results functionally link neural stem cell activity to specific patterns of gene expression.

Our previous study identified sfrp3 as a neuronal activity-regulated niche factor that exhibits control over multiple steps of adult hippocampal neurogenesis, including progenitor proliferation, newborn neuron maturation, dendritic growth and dendritic spine formation, as well as the activation of quiescent adult neural stem cells [32]. Sfrp3 is an essential mediator of some antidepressant actions in animal models and polymorphisms in the human gene are significantly associated with partial antidepressant responses in patients, which suggest that manipulation of SFRP3 action may represent a novel therapeutic approach to treat depression [40]. Here, we reveal higher expression levels of sfrp3 in the temporal hippocampus, which is the region more actively involved in mediating affective behaviors. Our study thus suggests how SFRP3 could be an efficacious and highly restricted extracellular target for fine-tuning adult hippocampal neurogenesis in a region-specific manner. Taken together, our results reveal a molecular gradient generated by local mature dentate granule neurons that couples adult neurogenesis to heterogeneities in the surrounding niche. This gradient provides a mechanism that may contribute to regional differences in adult neurogenesis and parallels the functional differences along the septotemporal axis. As several genes have recently been reported to be differentially expressed and regulated along the septo-temporal axis of the hippocampus [11, 15], sfrp3 may be one of multiple molecular gradients contributing to heterogeneous functional properties of the hippocampus through regulation of neural stem cell activation, neuronal development and circuit integration during adult neurogenesis.

\section{Conclusions}

We found that sfrp3, a secreted inhibitor of Wnt signaling, is expressed in a gradient along the septo-temporal axis of the dentate gyrus that is established during postnatal development. We provide functional evidence that this molecular gradient regulates quiescent RGL activation. Our study thus demonstrates, for the first time, a molecular niche mechanism to support region-specific differences in adult hippocampal neurogenesis along the longitudinal axis of hippocampus.

\section{Materials and methods}

Animals, housing, administration of tamoxifen and tissue processing

Eight-week-old mice of the following genotypes were used: wild-type (C57BL/6), sfrp3 wild-type (WT) and knockout (KO) (B6;129), nestin-CreER ${ }^{T 2+-} ; \mathrm{Z} / E G^{f /+}$; sfrp3 
-/- $(\mathrm{C} 57 \mathrm{BL} / 6)$ and nestin-CreER ${ }^{T 2+/-} ; \mathrm{Z} / E G^{f /+} ;$ sfrp3 ${ }^{+/+}$ $(\mathrm{C} 57 \mathrm{BL} / 6)$. Animals were housed in the standard facility. All animal procedures used in this study were performed in accordance with the protocol approved by the Institutional Animal Care and Use Committee.

A single low dose of tamoxifen $(62 \mathrm{mg} / \mathrm{kg}$ body weight, i.p.; Sigma) in 2-month-old mice resulted in sparse labeling at the clonal level for analysis at $7 \mathrm{dpi}$ in both WT and sfrp3 $\mathrm{KO}$ mice, as previously described [41]. Mice were anaesthetized $(100 \mu \mathrm{g}$ ketamine, $10 \mu \mathrm{g}$ xylazine in $10 \mu \mathrm{l}$ saline per gram) and perfused with $4 \%$ paraformaldehyde (PFA) and brains were postfixed in PFA overnight. Brains were then transferred to $30 \%$ sucrose and stored at $4{ }^{\circ} \mathrm{C}$ until brains sank to the bottom of tube.

\section{Anatomical definitions and sectioning}

For analysis of neurogenesis along the longitudinal axis, the right hippocampus was dissected and sectioned perpendicular to its long axis to enable comparable analyses along the entire axis. This axis is most precisely described as the septo-temporal axis (Fig. 1a). Sections were cut at $40 \mu \mathrm{m}$ using a cryostat (Leica CM 3050S) for a total of $\sim 80$ sections. Every sixth section was collected and processed for immunostaining.

For clonal analysis, coronal brain sections $(40 \mu \mathrm{m})$ through the entire dentate gyrus were collected in a serial order for a total of $\sim 50$ sections using a microtome (Leica SM 2010R), which is along the anterior-posterior axis.

\section{Immunostaining, confocal imaging, in situ hybridization and quantitative real-time reverse transcription PCR}

For immunostaining with anti-nestin and anti-MCM2, an antigen retrieval protocol was performed by microwaving sections in boiled citric buffer for $7 \mathrm{~min}$ as described previously [41]. Immunostaining was performed with the following primary antibodies: anti-GFP (Rockland; goat; 1:500 dilution), anti-nestin (Aves; chick; 1:500 dilution), anti-MCM2 (BD; mouse; 1:500 dilution). Images were acquired on a Zeiss LSM 710 confocal system (Carl Zeiss) with $\mathrm{a} \times 40$ objective lens using a multitrack configuration.

In situ hybridization analysis was performed similarly as previously described [60]. Briefly, $4 \%$ paraformamide-fixed cryo-protected brain tissue samples were embedded in O.C.T. mounting solution and frozen at $-80{ }^{\circ} \mathrm{C}$. Brain sections $(20 \mu \mathrm{m})$ were cut onto Superfrost-Plus slides (Fisher Scientific). Full-length digoxygenin-labeled antisense riboprobe for sfrp 3 was prepared by in vitro transcription. Sections were hybridized with the riboprobes at $65{ }^{\circ} \mathrm{C}$ overnight, and washed once in 5X SSC and $1 \%$ SDS, then twice in 2X SSC without SDS for $30 \mathrm{~min}$ each at $65{ }^{\circ} \mathrm{C}$. After overnight incubation with alkaline phosphataseconjugated anti-digoxygenin antibody at $4{ }^{\circ} \mathrm{C}$, hybridized riboprobes were visualized using nitro blue tetrazolium (NBT, $35 \mu \mathrm{g} / \mathrm{ml}$ )/5-bromo-4-chloro-3-indolyl phosphate (BCIP, $18 \mu \mathrm{g} / \mathrm{ml}$ ) color reaction at room temperature.

For quantitative real-time reverse transcription PCR, dentate gyrus tissue was rapidly micro-dissected from adult WT mice and cut into three fragments (septal, intermediate and temporal regions). For gene expression analysis, the total RNA fraction was immediately isolated after dissection (Qiagen), treated with DNAase and reverse-transcribed into the first-strand cDNA (Invitrogen). Specific primers as followed were used in SYBRgreen based quantitative real-time PCR to measure the expression level of target genes with the $\Delta \Delta \mathrm{Ct}$ method (ABI).

GAPDH: 5'- GTATTGGGCGCCTGGTCACC-3' (forward), 5'- CGCTCCTGGAAGATGGTGATGG-3' (reverse); sFRP3: 5'- CAAGGGACACCGTCAATCTT-3' (forward), 5'- CATATCCCAGCGCTTGACTT-3' (reverse).

\section{Quantification and statistical analysis}

For quantification of signal intensity of in situ hybridization on hippocampal coronal sections, brightfield images were taken at the same exposure and converted into grey scale. Granule cell layers of dentate gyrus were circled and mean grey values were obtained. For normalization, an area without signal was selected and mean grey values were calculated. The intensity indicated by the mean grey values in the granule cell layer after subtracting the mean grey value in the area without signal represents the relative expression levels. For quantification of signal intensity of in situ hybridization in hippocampal sagittal sections, brightfield images were manually segmented in LSM image browser (Zeiss) using the 'closed free shape curved drawing' and the 'extract region' tools. Segmented dentate gyri were then manually aligned using Reconstruct [45] and rotated such that the dentate gyrus occupied a maximum horizontal space. Aligned images were loaded as matrices into Matlab (Mathworks) for quantitative measurements. Matrices were summed across rows and the resulting vector was divided into 100 bins. The mean value for each bin was calculated and the resulting 100 mean values were used as the quantitative expression data across the dentate gyrus for each analyzed image.

For quantification of $\mathrm{MCM}^{+}$RGLs, an inverted ' $\mathrm{Y}$ ' shape from anti-nestin staining superimposed on MCM2 + nucleus was scored double positive for nestin and MCM2, as described previously [45]. All analyses were performed by investigators blind to experimental conditions. Statistical significance $(p<0.01)$ was assessed with a one-way ANOVA or Student's $t$ test, as indicated.

Competing interests

The authors declare that they have no competing interests. 


\section{Authors' contributions}

JS and MAB: conception and design, collection of data, and data analysis; JUG: conception and design; MHJ, HJ and BW: collection of data; GJS: quantification of in situ hybridization; ZY and KMC: design. GM and HS: conception and design, financial support, and manuscript writing. All authors read and approved the final manuscript.

\section{Acknowledgements}

We thank members of the Song and Ming laboratories for their suggestions, A. Rattner and J. Nathans for generating sfrp3 $\mathrm{KO}$ mice, K. Liu for help with in situ hybridization. This work was supported by the NIH (NS047344 and NS093772 to H.S; NS048271 and MH105128 to G.M.; MH090115 to M.-H.J.; and NS080913 to M.A.B) and NARSAD (to K.M.C., G.M. and H.S.).

\section{Author details}

${ }^{1}$ Tsinghua-Peking Joint Center for Life Sciences, Tsinghua University, Beijing 100084, P.R. China. ${ }^{2}$ Institute for Cell Engineering, Johns Hopkins University School of Medicine, Baltimore, MD 21205, USA. ${ }^{3}$ Department of Neurology, Johns Hopkins University School of Medicine, Baltimore, MD 21205, USA. ${ }^{4}$ Department of Neurologic Surgery, Department of Biochemistry and Molecular Biology, Mayo Clinic College of Medicine, Rochester, MN 55905, USA. ${ }^{5}$ The Solomon H. Snyder Department of Neuroscience, Johns Hopkins University School of Medicine, Baltimore, MD 21205, USA. ${ }^{6}$ Institutes of Brain Science and State Key Laboratory of Medical Neurobiology, Fudan University, Shanghai 200032, China. ${ }^{7}$ Department of Psychiatry and Behavioral Sciences, Johns Hopkins University School of Medicine, Baltimore, MD 21205, USA. ${ }^{8}$ Present Address: Broad CIRM Center and Department of Stem Cell Biology and Regenerative Medicine, Zilkha Neurogenetic Institute, University of Southern California Keck School of Medicine, Los Angeles, CA 90033, USA. ${ }^{9}$ Present Address: Whitehead Institute for Biomedical Research, Cambridge, MA 02142, USA.

\section{Received: 22 February 2015 Accepted: 25 August 2015} Published online: 04 September 2015

\section{References}

1. Bannerman DM, Grubb M, Deacon RM, Yee BK, Feldon J, Rawlins JN. Ventral hippocampal lesions affect anxiety but not spatial learning. Behav Brain Res. 2003;139(1-2):197-213.

2. Moser MB, Moser El. Functional differentiation in the hippocampus. Hippocampus. 1998;8(6):608-19.

3. Amaral DG, Witter MP. The three-dimensional organization of the hippocampal formation: a review of anatomical data. Neuroscience. 1989;31(3):571-91.

4. Jinno S. Topographic differences in adult neurogenesis in the mouse hippocampus: a stereology-based study using endogenous markers. Hippocampus. 2011;21(5):467-80.

5. Cenquizca LA, Swanson LW. Spatial organization of direct hippocampal field CA1 axonal projections to the rest of the cerebral cortex. Brain Res Rev. 2007:56(1):1-26.

6. Dolorfo CL, Amaral DG. Entorhinal cortex of the rat: topographic organization of the cells of origin of the perforant path projection to the dentate gyrus. J Comp Neurol. 1998;398(1):25-48.

7. Gage FH, Thompson RG. Differential distribution of norepinephrine and serotonin along the dorsal-ventral axis of the hippocampal formation. Brain Res Bull. 1980;5(6):771-3.

8. Jinno S, Kosaka T. Cellular architecture of the mouse hippocampus: a quantitative aspect of chemically defined GABAergic neurons with stereology. Neurosci Res. 2006;56(3):229-45.

9. Jung MW, Wiener SI, McNaughton BL. Comparison of spatial firing characteristics of units in dorsal and ventral hippocampus of the rat. J Neurosci. 1994;14(12):7347-56

10. Tanaka KF, Samuels BA, Hen R. Serotonin receptor expression along the dorsal-ventral axis of mouse hippocampus. Philos Trans R Soc Lond B Biol Sci. 2012;367(1601):2395-401.

11. Thompson CL, Pathak SD, Jeromin A, Ng LL, MacPherson CR, Mortrud MT, et al. Genomic anatomy of the hippocampus. Neuron. 2008;60(6):1010-21.

12. Bannerman DM, Deacon RM, Offen S, Friswell J, Grubb M, Rawlins JN. Double dissociation of function within the hippocampus: spatial memory and hyponeophagia. Behav Neurosci. 2002;116(5):884-901.
13. Bannerman DM, Rawlins JN, McHugh SB, Deacon RM, Yee BK, Bast T, et al. Regional dissociations within the hippocampus-memory and anxiety. Neurosci Biobehav Rev. 2004;28(3):273-83.

14. Kheirbek MA, Drew LJ, Burghardt NS, Costantini DO, Tannenholz L, Ahmari $\mathrm{SE}$, et al. Differential control of learning and anxiety along the dorsoventral axis of the dentate gyrus. Neuron. 2013;77(5):955-68.

15. Christensen T, Bisgaard CF, Nielsen HB, Wiborg O. Transcriptome differentiation along the dorso-ventral axis in laser-captured microdissected rat hippocampal granular cell layer. Neuroscience. 2010;170(3):731-41.

16. Ming GL, Song H. Adult neurogenesis in the mammalian brain: significant answers and significant questions. Neuron. 2011;70(4):687-702.

17. Braun $\mathrm{SMG}$, Jessberger $\mathrm{S}$. Adult neurogenesis in the mammalian brain. Front Biol. 2013;8(3):295-304

18. Christian KM, Song H, Ming GL. Functions and dysfunctions of adult hippocampal neurogenesis. Annu Rev Neurosci. 2014;37:243-62.

19. Bonaguidi MA, Song J, Ming GL, Song H. A unifying hypothesis on mammalian neural stem cell properties in the adult hippocampus. Curr Opin Neurobiol. 2012;22(5):754-61.

20. Snyder JS, Radik R, Wojtowicz JM, Cameron HA. Anatomical gradients of adult neurogenesis and activity: young neurons in the ventral dentate gyrus are activated by water maze training. Hippocampus. 2009;19(4):360-70.

21. Jinno S. Topographic differences in adult neurogenesis in the mouse hippocampus: A stereology-based study using endogenous markers. Hippocampus. 2011;21(5):467-80.

22. Piatti VC, Davies-Sala MG, Esposito MS, Mongiat LA, Trinchero MF, Schinder AF. The timing for neuronal maturation in the adult hippocampus is modulated by local network activity. J Neurosci. 2011;31(21):7715-28.

23. Jinno $\mathrm{S}$. Decline in adult neurogenesis during aging follows a topographic pattern in the mouse hippocampus. J Comp Neurol. 2011;519(3):451-66.

24. Snyder JS, Ferrante SC, Cameron HA. Late maturation of adult-born neurons in the temporal dentate gyrus. PLoS One. 2012;7(11), e48757.

25. Wu MV, Hen R. Functional dissociation of adult-born neurons along the dorsoventral axis of the dentate gyrus. Hippocampus. 2014;24(7):751-61.

26. Schwank G, Basler K. Regulation of organ growth by morphogen gradients. Cold Spring Harb Perspect Biol. 2010;2(1):a001669.

27. Ciani L, Salinas PC. WNTs in the vertebrate nervous system: from patterning to neuronal connectivity. Nat Rev Neurosci. 2005;6(5):351-62.

28. Harrison-Uy SJ, Pleasure SJ. Wnt signaling and forebrain development. Cold Spring Harb Perspect Biol. 2012;4(7):a008094.

29. Lee SM, Tole S, Grove E, McMahon AP. A local Wnt-3a signal is required for development of the mammalian hippocampus. Development. 2000;127(3):457-67.

30. Machon O, Backman M, Machonova O, Kozmik Z, Vacik T, Andersen L, et al. A dynamic gradient of Wnt signaling controls initiation of neurogenesis in the mammalian cortex and cellular specification in the hippocampus. Dev Biol. 2007;311(1):223-37.

31. Shimogori T, VanSant J, Paik E, Grove EA. Members of the Wnt, Fz, and Frp gene families expressed in postnatal mouse cerebral cortex. J Comp Neurol. 2004;473(4):496-510.

32. Jang MH, Bonaguidi MA, Kitabatake Y, Sun J, Song J, Kang E, et al. Secreted frizzled-related protein 3 regulates activity-dependent adult hippocampal neurogenesis. Cell Stem Cell. 2013;12(2):215-23.

33. Seib DR, Corsini NS, Ellwanger K, Plaas C, Mateos A, Pitzer C, et al. Loss of Dickkopf-1 restores neurogenesis in old age and counteracts cognitive decline. Cell Stem Cell. 2013;12(2):204-14.

34. Lie DC, Colamarino SA, Song HJ, Desire L, Mira H, Consiglio A, et al. Wnt signalling regulates adult hippocampal neurogenesis. Nature. 2005:437(7063):1370-5

35. Qu Q, Sun G, Murai K, Ye P, Li W, Asuelime G, et al. Wnt7a regulates multiple steps of neurogenesis. Mol Cell Biol. 2013;33(13):2551-9.

36. Miranda CJ, Braun L, Jiang Y, Hester ME, Zhang L, Riolo M, et al. Aging brain microenvironment decreases hippocampal neurogenesis through Wntmediated survivin signaling. Aging Cell. 2012;11(3):542-52.

37. Chen M, Do H. Wnt Signaling in Neurogenesis during Aging and Physical Activity. Brain Sci. 2012;2(4):745-68.

38. Varela-Nallar $\mathrm{L}$, Inestrosa NC. Wnt signaling in the regulation of adult hippocampal neurogenesis. Front Cell Neurosci. 2013;7:100.

39. Faigle $\mathrm{R}$, Song $\mathrm{H}$. Signaling mechanisms regulating adult neural stem cells and neurogenesis. Biochim Biophys Acta. 2013;1830(2):2435-48. 
40. Jang MH, Kitabatake $Y$, Kang E, Jun H, Pletnikov MV, Christian KM, et al Secreted frizzled-related protein 3 (SFRP3) regulates antidepressant responses in mice and humans. Mol Psychiatry. 2013;18(9):957-8.

41. Bonaguidi MA, Wheeler MA, Shapiro JS, Stadel RP, Sun GJ, Ming GL, et al. In vivo clonal analysis reveals self-renewing and multipotent adult neural stem cell characteristics. Cell. 2011;145(7):1142-55.

42. Frade JM, Ovejero-Benito MC. Neuronal cell cycle: the neuron itself and its circumstances. Cell Cycle. 2015;14(5):712-20.

43. Maiorano D, Lutzmann M, Mechali M. MCM proteins and DNA replication. Curr Opin Cell Biol. 2006;18(2):130-6.

44. Maslov AY, Barone TA, Plunkett RJ, Pruitt SC. Neural stem cell detection, characterization, and age-related changes in the subventricular zone of mice. J Neurosci. 2004:24(7):1726-33.

45. Song J, Zhong C, Bonaguidi MA, Sun GJ, Hsu D, Gu Y, et al. Neuronal circuitry mechanism regulating adult quiescent neural stem-cell fate decision. Nature. 2012;489(7414):150-4.

46. Tye BK. MCM proteins in DNA replication. Annu Rev Biochem. 1999:68:649-86.

47. Lein ES, Zhao X, Gage FH. Defining a molecular atlas of the hippocampus using DNA microarrays and high-throughput in situ hybridization. J Neurosci. 2004;24(15):3879-89.

48. Mangale VS, Hirokawa KE, Satyaki PR, Gokulchandran N, Chikbire S, Subramanian L, et al. Lhx2 selector activity specifies cortical identity and suppresses hippocampal organizer fate. Science. 2008;319(5861):304-9.

49. Kim AS, Lowenstein DH, Pleasure SJ. Wnt receptors and Wnt inhibitors are expressed in gradients in the developing telencephalon. Mech Dev. 2001;103(1-2):167-72.

50. Green D, Whitener AE, Mohanty S, Lekven AC. Vertebrate nervous system posteriorization: Grading the function of Wnt signaling. Dev Dyn. 2015;244(3):507-12.

51. Grove EA, Tole S, Limon J, Yip L, Ragsdale CW. The hem of the embryonic cerebral cortex is defined by the expression of multiple Wnt genes and is compromised in Gli3-deficient mice. Development. 1998;125(12):2315-25.

52. Sahay A, Hen R. Adult hippocampal neurogenesis in depression. Nat Neurosci. 2007;10(9):1110-5.

53. Sahay A, Scobie KN, Hill AS, O'Carroll CM, Kheirbek MA, Burghardt NS, et al. Increasing adult hippocampal neurogenesis is sufficient to improve pattern separation. Nature. 2011;472(7344):466-70.

54. Snyder JS, Soumier A, Brewer M, Pickel J, Cameron HA. Adult hippocampal neurogenesis buffers stress responses and depressive behaviour. Nature. 2011;476(7361):458-61.

55. Stone SS, Teixeira CM, Devito LM, Zaslavsky K, Josselyn SA, Lozano AM, et al. Stimulation of entorhinal cortex promotes adult neurogenesis and facilitates spatial memory. J Neurosci. 2011;31(38):13469-84.

56. Deng W, Aimone JB, Gage FH. New neurons and new memories: how does adult hippocampal neurogenesis affect learning and memory? Nat Rev Neurosci. 2010;11(5):339-50.

57. Kitabatake $Y$, Sailor KA, Ming GL, Song H. Adult neurogenesis and hippocampal memory function: new cells, more plasticity, new memories? Neurosurg Clin N Am. 2007;18(1):105-13.

58. Bjarkam CR, Sorensen JC, Geneser FA. Distribution and morphology of serotonin-immunoreactive axons in the hippocampal region of the New Zealand white rabbit. I. Area dentata and hippocampus. Hippocampus. 2003;13(1):21-37.

59. Maggio N, Segal M. Unique regulation of long term potentiation in the rat ventral hippocampus. Hippocampus. 2007;17(1):10-25.

60. Ma DK, Jang MH, Guo JU, Kitabatake Y, Chang ML, Pow-Anpongkul N, et al. Neuronal activity-induced Gadd45b promotes epigenetic DNA demethylation and adult neurogenesis. Science. 2009;323(5917):1074-7.

\section{Submit your next manuscript to BioMed Central and take full advantage of:}

- Convenient online submission

- Thorough peer review

- No space constraints or color figure charges

- Immediate publication on acceptance

- Inclusion in PubMed, CAS, Scopus and Google Scholar

- Research which is freely available for redistribution

Submit your manuscript at www.biomedcentral.com/submit 